Małgorzata Spychała-Wawrzyniak Uniwersytet im. Adama Mickiewicza w Poznaniu malgorzata.spychala@amu.edu.pl

\title{
Situación laboral y cualificaciones profesionales de los profesores de español como lengua extranjera (ELE) en las escuelas secundarias de Polonia
}

\begin{abstract}
Resumen:
El objetivo de este artículo es presentar la situación profesional y las cualificaciones de los profesores de ELE. Queremos averiguar cuál es la formación de los profesores que trabajan en las escuelas polacas y si se sienten preparados para ejercer esta labor. Partimos de la tesis de que los profesores de ELE no están suficientemente preparados debido a la falta de formadores cualificados de ELE en las universidades polacas y a la programación de la formación didáctica, que omite contenidos relacionados especialmente con la enseñanza de lenguas extranjeras. También nos interesa saber qué motivó a los docentes que trabajan en las escuelas secundarias a convertirse en profesores de ELE y si están satisfechos con su trabajo. La metodología de esta investigación se basa en el análisis de las encuestas realizadas entre los profesores de ELE de las escuelas secundarias en Polonia.
\end{abstract}

Palabras clave: formación de profesores, cualificaciones, competencias, enseñanza de ELE, satisfacción laboral
Abstract:
Professional Status and Qualifications of Spanish Language Teachers in Se- condary Schools in Poland
The goal of this paper is to present the professional status and qualifications of Spanish language teachers. We want to verify if the teachers employed in 
secondary schools in Poland possess adequate qualifications and competence, and whether they are professionally prepared for their task. Our preliminary assumption is that Spanish language teachers do not feel adequately prepared for school work. This may be cased by a shortage of experts in teaching Spanish in Polish secondary schools, and the current education standards for teachers, which practically ignore the specific issues of foreign language teaching. We also are interested in examining the reasons that made teachers choose this profession and their satisfaction with this choice. The methodology of the survey conducted is based on the analysis of online questionnaires conducted among secondary school teachers in Poland.

Keywords: teacher education, professional qualifications, skills, Spanish language teaching, job satisfaction

\section{La problemática de la situación laboral docente}

Durante el proceso de aprendizaje de una lengua extranjera, la figura del profesor, su formación, sus habilidades y actitudes pueden favorecer y crear un clima adecuado de enseñanza. Sin duda, la calidad educativa se debe también a la satisfacción laboral de los docentes.

La satisfacción laboral puede ser definida como una actitud o conjunto de actitudes desarrolladas hacia la situación laboral. En general, se trata de emociones o actitudes positivas hacia el trabajo (Bravo, Peiró, Rodríguez, 1996; Anaya Nieto, Suárez Riveiro, 2007). Chiang Vega, Martín Rodrigo y Núñez Partido (2010: 157) sostienen que, en este caso, las actitudes "podrían pensarse como partículas psíquicas de una red que se ha ido formando a lo largo de la historia interactiva de cada individuo con su ambiente social". Hermosa Rodríguez (2006) añade que las actitudes tanto positivas como negativas pueden ser una fuente de satisfacción laboral o una causa del síndrome de burnout ("quemarse por el trabajo"), que pueden influir en el proceso de enseñanza.

Para medir la satisfacción laboral global de los docentes, Anaya Nieto y Suárez Riveiro (2007: 223) proponen analizar sobre todo las siguientes variables morales: el gusto por el trabajo actual, el gusto 
por la vida en el centro del trabajo, el deseo de cambio laboral y el deseo de jubilación anticipada (las dos primeras cuestiones se asocian más con una mayor satisfacción y las dos últimas muestran una baja satisfacción laboral). Los autores han elaborado un modelo de satisfacción laboral de los profesores, que se compone de varias facetas, que han sido estructuradas en cinco dimensiones: el diseño de trabajo, las condiciones de vida asociadas al trabajo, la realización personal, la promoción y las relaciones con los superiores y el salario ${ }^{1}$.

Zabalza Beraza y Zabalza Cerdeiriña (2012: 60), basándose en varias investigaciones acerca de la práctica laboral de los docentes, recuerdan algunos núcleos de satisfacción e insatisfacción personal y profesional. Entre los factores de satisfacción laboral, los profesores señalaban la posibilidad de estar en contacto con niños. "Estar con los niños significaba, en lo operativo, actuar como compañía adulta pero también como instructor y como agente disciplinario." Entre las insatisfacciones se enumeraban especialmente los problemas administrativos y burocráticos, ya que los profesores sienten "que se trata de actividades que les alejan de su interés fundamental que es estar con los niños".

Los profesores de lenguas también pueden sentirse frustrados debido a una baja remuneración de su trabajo, la brevedad de los contratos, etc.; sin embargo, muchos de ellos continúan trabajando teniendo en cuenta otras características de esta profesión: el horario flexible, la autonomía en la clase, el progreso lingüístico de los alumnos, el poder enseñar la cultura, etc. ${ }^{2}$ (Senior, 2006).

A continuación, en nuestra investigación, vamos a analizar qué importancia dan los profesores de ELE en Polonia a factores como: el contacto con los alumnos, el horario, el salario, etc. Analizaremos también su formación y sus actitudes hacia esta labor.

${ }^{1}$ En nuestra investigación analizamos especialmente las condiciones de vida asociadas al trabajo, el salario y también el deseo de cambio laboral.

2 Senior (2006: 243) habla de "satisfacción intercultural" que consiste en interesarse por otras personas y sus culturas, como también el poder ayudar a los inmigrantes. La autora recuerda que en muchas ocasiones el profesor de lenguas se encuentra en la clase con algo llamado "microcosmos de la sociedad". 


\section{La enseñanza de ELE y la formación de los profesores en Polonia}

Antes de describir brevemente el modelo de formación de los profesores de lenguas extranjeras en Polonia, queremos presentar la situación del español en las escuelas polacas, según los datos del Ministerio de Educación (2016). En el año escolar 2016-2017 el español como lengua obligatoria fue estudiado por 99.644 alumnos, y como lengua adicional, por 23.015. En el esquema (Fig. 1) presentamos la distribución de la enseñanza de las lenguas extranjeras obligatorias en comparación con el español en los años escolares 2015-2016 y 2016-2017. Como podemos ver, el español ocupa el cuarto lugar, después del inglés, el alemán, el ruso y el francés en cuanto al número de estudiantes. Sin embargo, hay que subrayar que, mientras que el número de estudiantes de español ha crecido significativamente, el número de estudiantes de alemán y ruso ha disminuido ligeramente desde el curso escolar 2015-2016.

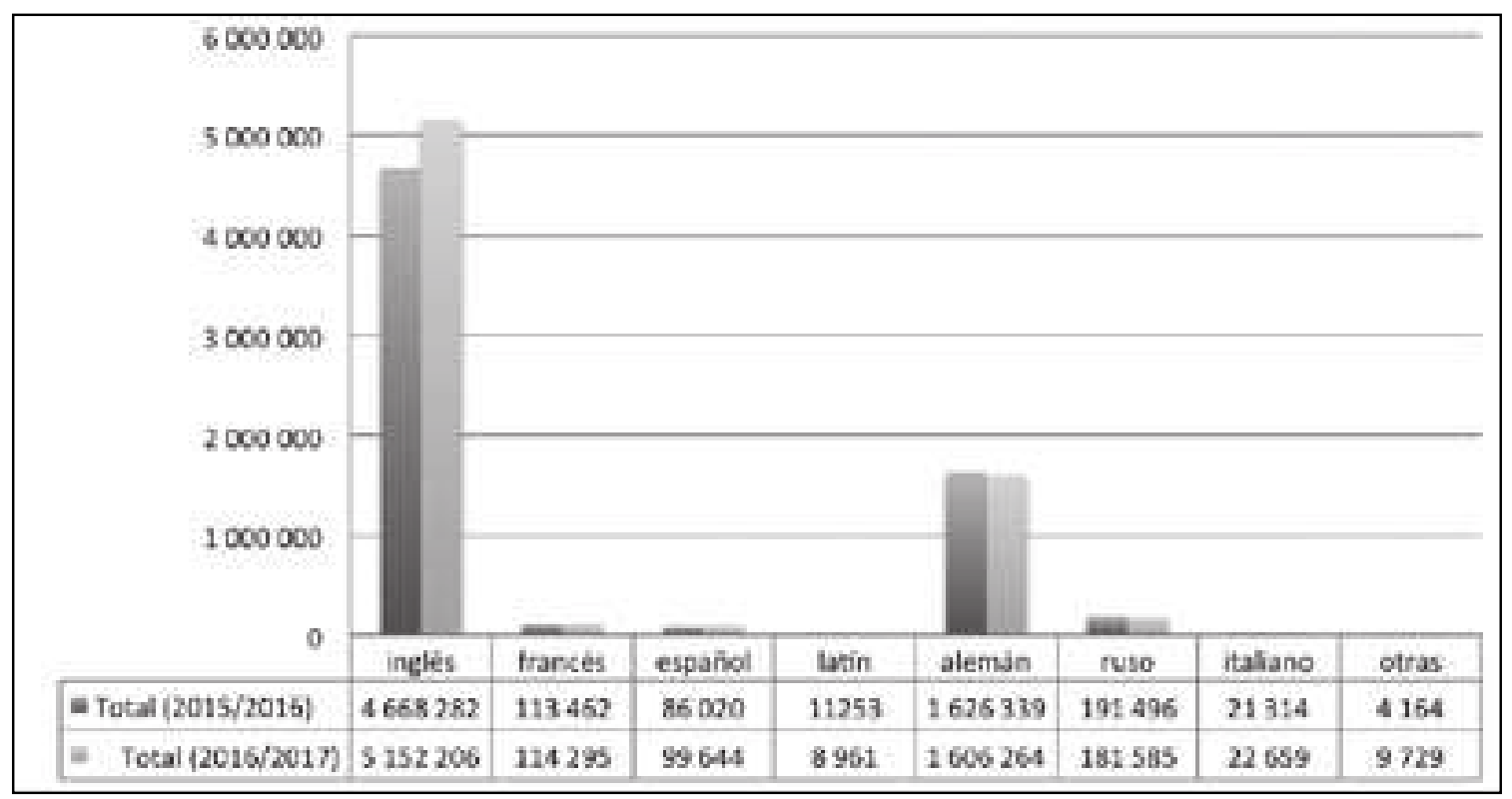

Fig. 1. Enseñanza de lenguas extranjeras obligatorias en Polonia en los años escolares 2015- 2016 y 2016-2017. Elaboración propia a partir de los datos del Ministerio de Educación (2016). 
Sin duda, en los últimos años el español ha experimentado el mayor crecimiento en las escuelas preuniversitarias polacas, como demuestran los datos presentados en la Fig. 2.

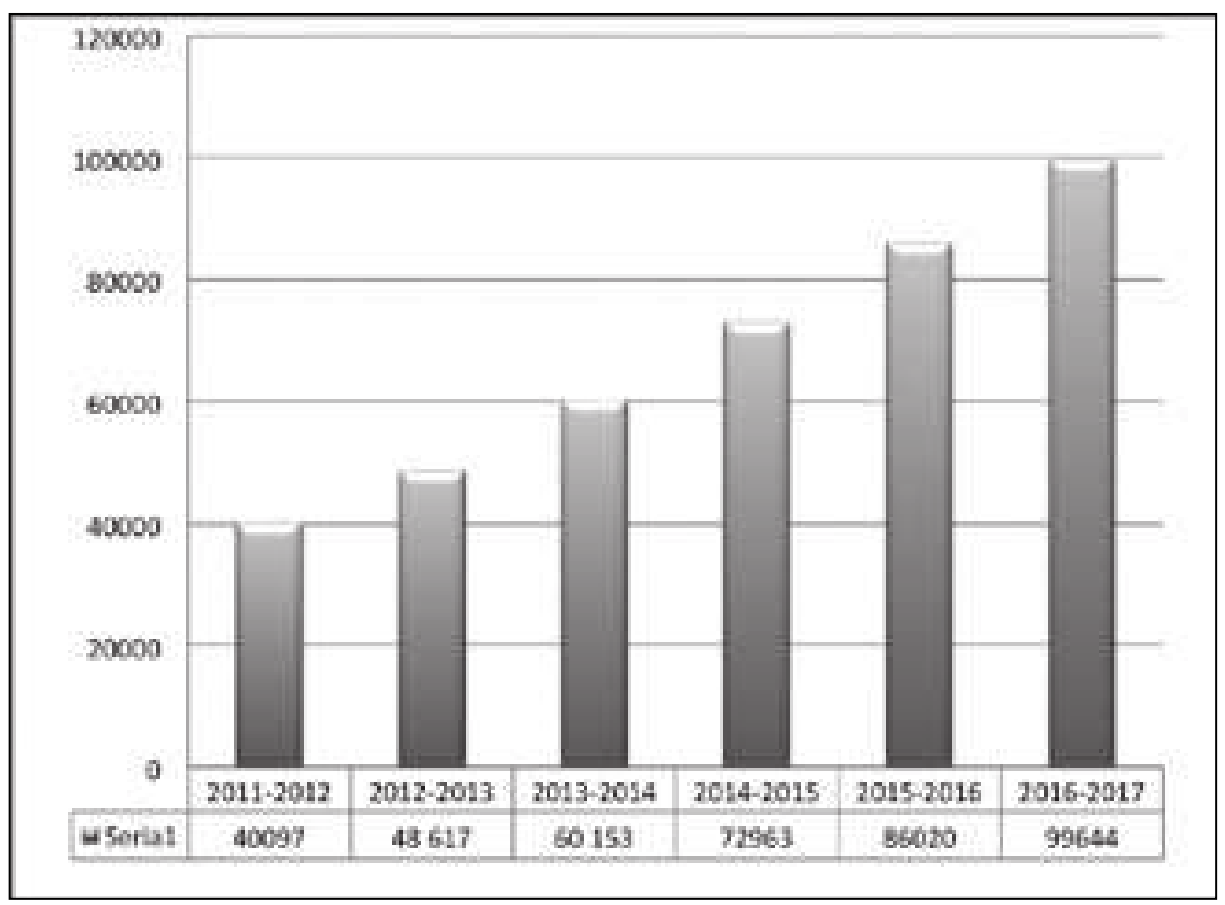

Fig. 2. Enseñanza del español como lengua obligatoria en las escuelas preuniversitarias en Polonia en los años 2011-2017

En cuanto a la enseñanza de ELE como lengua obligatoria en el curso escolar 2016-2017, según distintos tipos de escuelas, casi la mitad de los alumnos eran de liceo (45.924) y otros tantos (39.261) eran estudiantes de educación secundaria obligatoria (gimnazjum). Debemos apuntar también que desde el año 2015 ha crecido el número del alumnado de español en la escuela primaria. En el curso escolar 20152016 había 6343 alumnos y en el curso 2016-2017, 6891 alumnos $^{3}$.

${ }^{3}$ Debemos señalar que la situación del español puede cambiar con la nueva reforma de educación en Polonia, que empezará con el curso 2017-2018. El objetivo de la reforma es eliminar la actual educación secundaria obligatoria (gimnazjum), para formar ocho años de escuela primaria y cuatro años de secundaria. En esta situación lo más probable será que el español pase a las dos últimas clases de la escuela primaria. 
El análisis de estos datos nos permite establecer la hipótesis de que el número de profesores de español también crece de manera significativa.

$\mathrm{Si}$ alguien quiere trabajar como profesor de una lengua extranjera en una escuela preuniversitaria en Polonia, debe disponer de una formación especializada ${ }^{4}$. La programación de esta especialización didáctica es bastante compleja, ya que exige completar una preparación en Psicología y Pedagogía (90+60 horas) y una preparación didáctica $(30+90 \text { horas })^{5}$. Además, el candidato al puesto de profesor debe aprobar las siguientes asignaturas: Uso de las $\mathrm{TIC}^{6}$, Higiene vocal e Higiene del trabajo (10-30 horas). Cada candidato también debe realizar en total 150 horas de prácticas en una escuela, de las cuales 30 horas se dedican expresamente a la práctica pedagógica.

\section{Los profesores de ELE en Polonia. Metodología de la investigación}

La metodología de esta investigación ha sido empírica (cuantitativa y cualitativa). Se han enviado a todos los profesores que colaboraron en las Olimpiadas de ELE de 2015-2016 cuestionarios por correo electrónico ${ }^{7}$. El objetivo de los cuestionarios era conocer las opiniones de los profesores de ELE que trabajaban en las escuelas polacas preuniversitarias sobre las condiciones en las que se desarrollaba su profesión. La encuesta se llevó a cabo durante el primer semestre del curso 2015-2016. En varias preguntas se utilizó la escala de Likert

${ }^{4}$ Actualmente las normas de formación de los profesores las describe la ley del Ministerio de Ciencia y Enseñanza Superior, de 17 de enero de 2012 (Dz. U. 2012, poz. 131).

${ }^{5}$ La programación de la preparación didáctica incluye 30 horas dedicadas a nociones generales de didáctica general y 90 horas de didáctica de una materia especializada, en nuestro caso sería la didáctica de ELE. Se supone que las 90 horas de didáctica se imparten en la lengua meta. En cuanto a la preparación psicológica y pedagógica, 90 horas se dedican a nociones generales y 60 horas a la preparación relacionada con la edad de los alumnos.

${ }^{6}$ Las tecnologías de la información y la comunicación.

${ }^{7}$ La encuesta fue realizada con el apoyo del Comité Organizador de las Olimpiadas Nacionales de Español en Polonia. 
(de cinco valores: $1=$ Muy bajo; $2=$ Bajo; $3=$ Medio; $4=$ Alto; 5=Muy alto) para conocer las distintas valoraciones de los encuestados. Nos planteamos varias preguntas de investigación (Anexo 1). Entre las preguntas generales se encuentran las siguientes:

- ¿Cuál es la formación de los profesores que imparten español en las escuelas polacas? ¿Disponen estos de las cualificaciones ministeriales?

- ¿Por qué han elegido la profesión de profesor de ELE?

Respondieron 128 profesores (114 mujeres y 14 hombres $)^{8}$. La mayoría de ellos son jóvenes (entre 25-35 años), profesores contratados (nauczyciel kontraktowy) ${ }^{9}$ y de nacionalidad polaca. 120 de ellos tienen contrato fijo con la escuela y 77 son profesores de liceo. En la tabla 1 presentamos todos los datos de la muestra.

\begin{tabular}{|c|c|}
\hline \multicolumn{2}{|c|}{ VARIABLES DESCRIPTIVAS - 128 profesores } \\
\hline Sexo & $\begin{array}{ll}\text { - } & \text { Hombre }-14 \\
\text { - } & \text { Mujer }-114\end{array}$ \\
\hline Nacionalidad & $\begin{array}{ll}\text { - } & \text { polaco/a }-115 \\
\text { - } & \text { español/a }-8 \\
\text { - } & \text { argentino/a }-1 \\
\text { - } & \text { boliviano/a }-1 \\
\text { - } & \text { cominicano/a }-1 \\
\text { - } & \text { venezolano/a }-1 \\
& \end{array}$ \\
\hline
\end{tabular}

${ }^{8}$ Se envío el cuestionario a los 437 profesores que colaboraban entonces con las Olimpiadas de Español.

${ }^{9}$ En la trayectoria profesional de los profesores de enseñanza primaria y secundaria en Polonia, el puesto del profesor contratado ocupa el segundo lugar en la carrera académica. En total hay 4 grados de esta carrera: profesor en prácticas, profesor contratado, profesor designado y profesor diplomado. 


\begin{tabular}{|c|c|}
\hline \multicolumn{2}{|c|}{ VARIABLES DESCRIPTIVAS - 128 profesores } \\
\hline Edad & $\begin{array}{ll}\text { - } & \text { Menos de } 25 \text { años }-1 \\
\text { - } & \text { Entre } 25 \text { y } 35 \text { años }-63 \\
\text { - } & \text { Entre } 36 \text { y } 45 \text { años }-45 \\
\text { - } & \text { Entre } 46 \text { y } 55 \text { años }-15 \\
\text { - } & \text { Entre } 56 \text { y } 65 \text { años }-4\end{array}$ \\
\hline $\begin{array}{l}\text { Primer centro de } \\
\text { trabajo }\end{array}$ & $\begin{array}{l}\text { - Szkoła podstawowa (primaria) -6 } \\
\text { - Gimnazjum (secundaria obligatoria) } \\
-33 \\
\text { - Liceum (bachillerato) - } 77 \\
\text { - Technikum (escuela de formación téc- } \\
\text { nica) - } 3 \\
\text { - Szkoła zawodowa (escuela de forma- } \\
\text { ción profesional) - } 1 \\
\text { - Universidad o escuela superior }-5 \\
\text { - Otro - } 3\end{array}$ \\
\hline $\begin{array}{l}\text { Trabaja en un Centro } \\
\text { Bilingüe }\end{array}$ & $\begin{array}{ll}\text { - } & \text { Sí }-26 \\
\text { - } & \text { No }-102\end{array}$ \\
\hline Tiene contrato fijo & $\begin{array}{l}\text { - } \quad \text { Sí - } 110 \\
\text { - } \mathrm{No}-18\end{array}$ \\
\hline Situación laboral & $\begin{array}{l}\text { - } \quad \text { Nauczyciel stażysta (en prácticas) - } 9 \\
\text { - } \quad \text { Nauczyciel kontraktowy (contratado) } \\
-58 \\
\text { - } \quad \text { Nauczyciel mianowany (nombrado) } \\
-26 \\
\text { - } \quad \text { Nauczyciel dyplomowany (diplomado) } \\
-29 \\
\text { - Otra }-6\end{array}$ \\
\hline
\end{tabular}

Tabla 1. Distribución de las variables 
En el siguiente gráfico (Fig. 3) podemos ver que más de la mitad de los profesores no dispone de mucha experiencia laboral; 31 profesores tienen menos de 5 años de experiencia, 47 han declarado que tienen entre 5 y 10 años de experiencia. Esta situación se debe especialmente a la escasa presencia del español en las escuelas polacas antes del año 2000 .

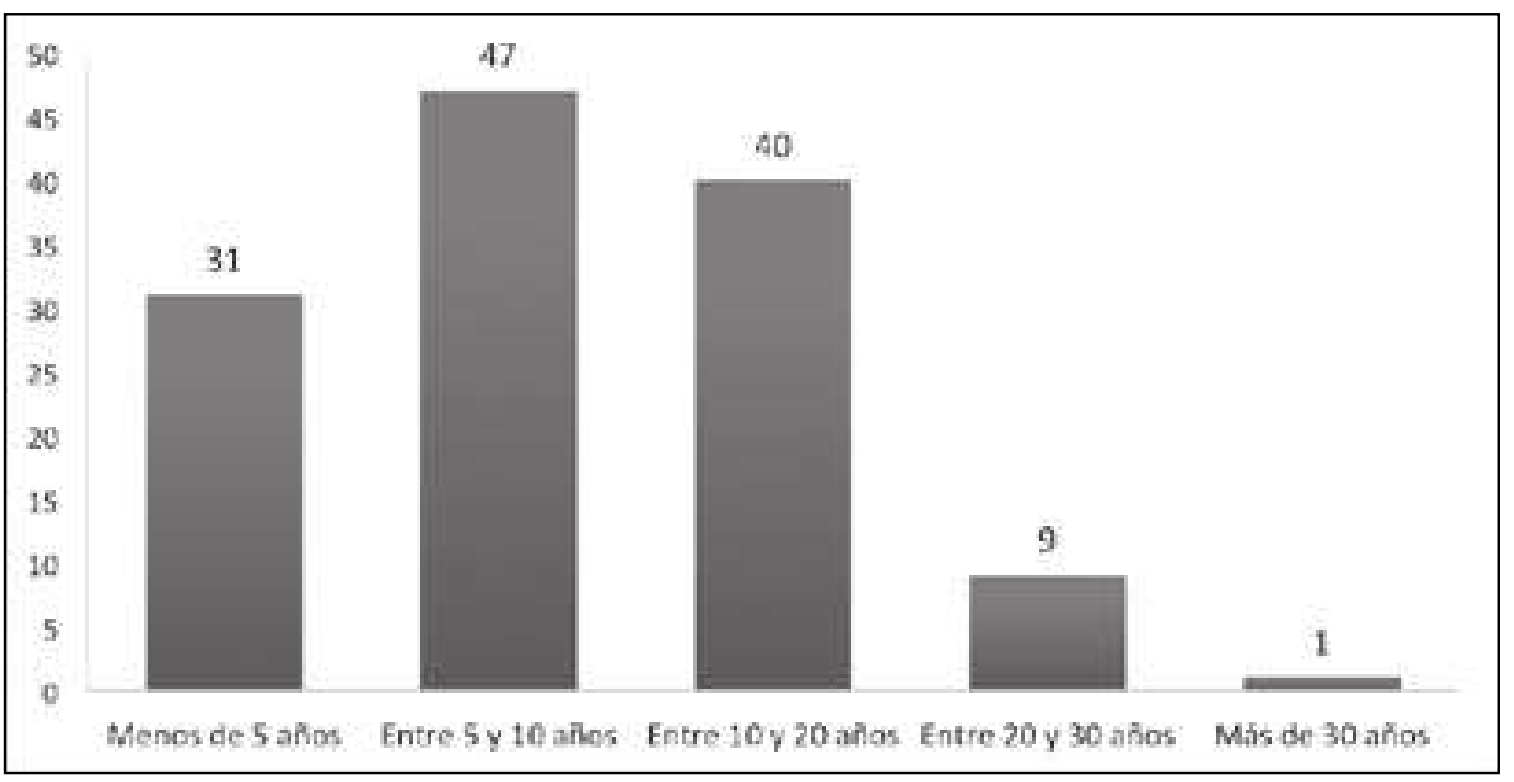

Fig. 3. Años de experiencia laboral como profesor/a de ELE $(n=128)$

Nos interesaba también averiguar por qué los docentes han decidido ser profesores de ELE (Fig. 4). Verificamos que casi la mitad de los encuestados (47\%) indica que recuerda haber querido siempre ejercer esta profesión, mientras que el $23 \%$ de ellos son profesores de otra asignatura y decidieron ampliar su formación. El 16\% ha señalado "otro motivo", mientras que el 9\% no ha conseguido trabajo en otro ámbito profesional y por eso han decidido ser profesores de español. El 5\% de los encuestados ha señalado que alguien los convenció para realizar la especialización didáctica durante los estudios universitarios. 


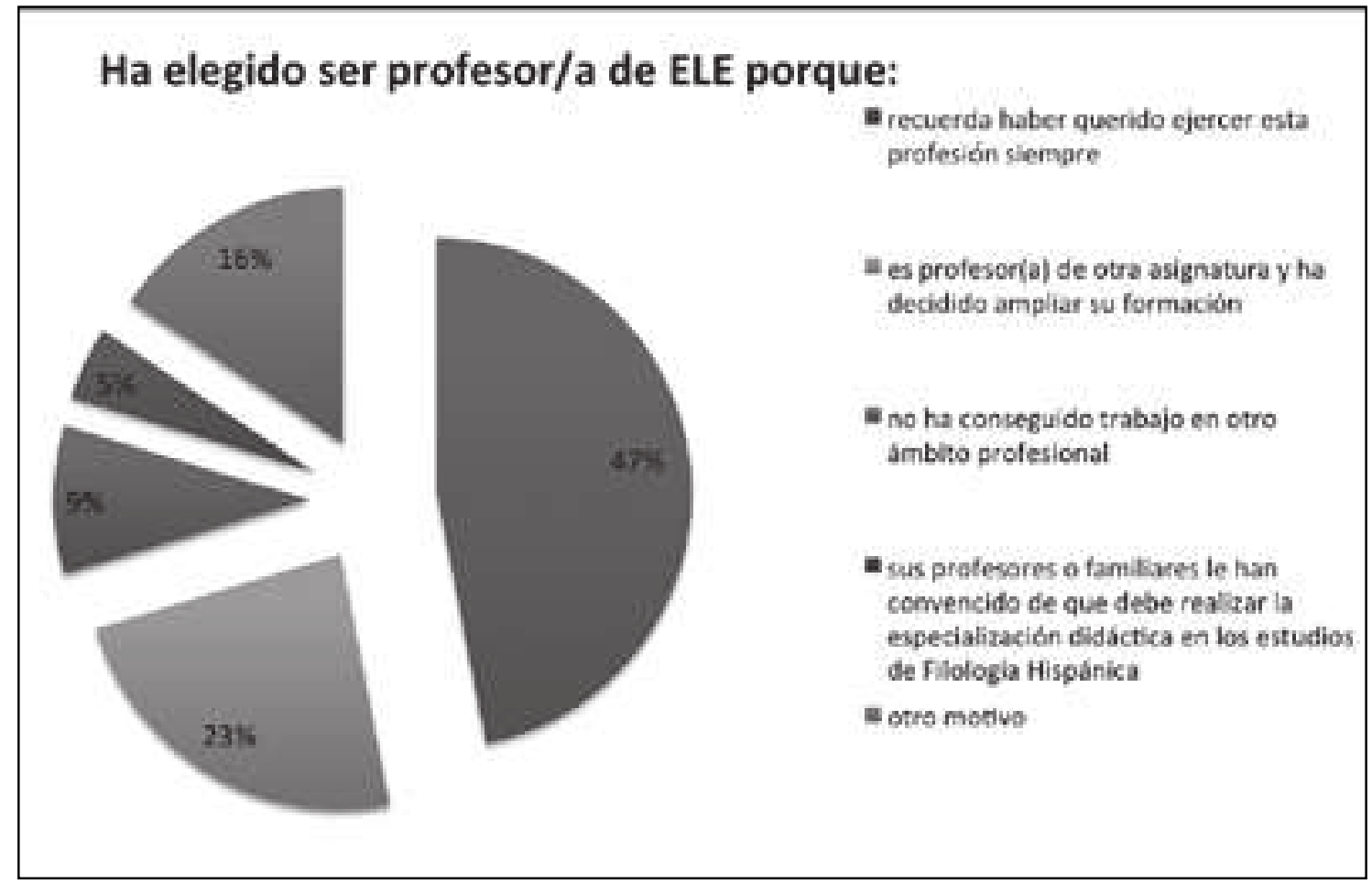

Fig. 4. Motivos para elegir la profesión de profesor de ELE

En cuanto a las cualificaciones exigidas a los profesores de ELE para poder trabajar en escuelas preuniversitarias (Fig. 5), la mayoría de los participantes de la encuesta $(89 \%)$ ha confirmado que dispone de ellas. Este resultado sorprende de manera positiva, teniendo en cuenta la poca oferta de especialización didáctica dedicada a la enseñanza de ELE en las universidades polacas. Como podemos ver en el siguiente gráfico (Fig. 6), el 68\% de los profesores ha realizado la especialización en los estudios de Filología Hispánica; de ellos, el 34\% la completó porque era consciente de su importancia para su futuro profesional. 
¿Dispone de las cualificaciones (incluyendo la relativa a la didáctica de ELE) que exige legislación polaca?

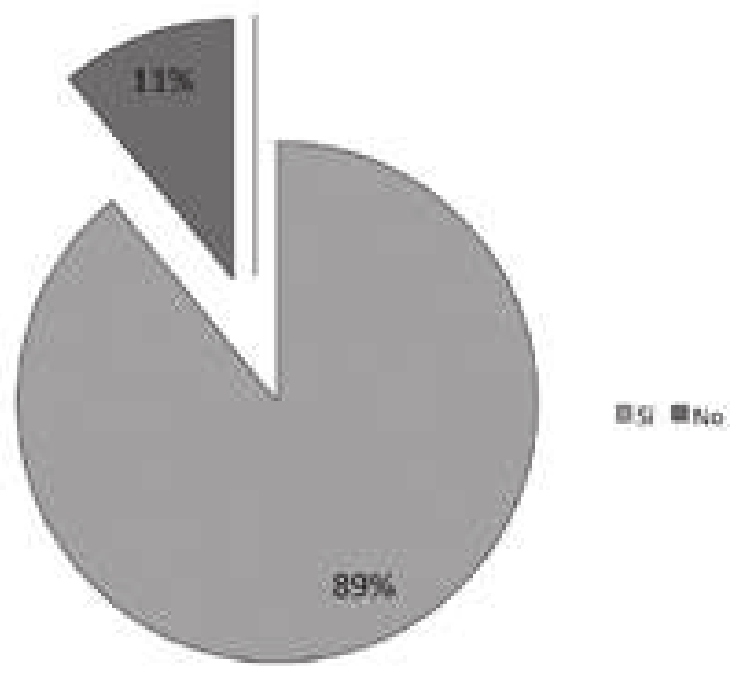

Fig. 5. Distribución de los profesores según sus cualificaciones

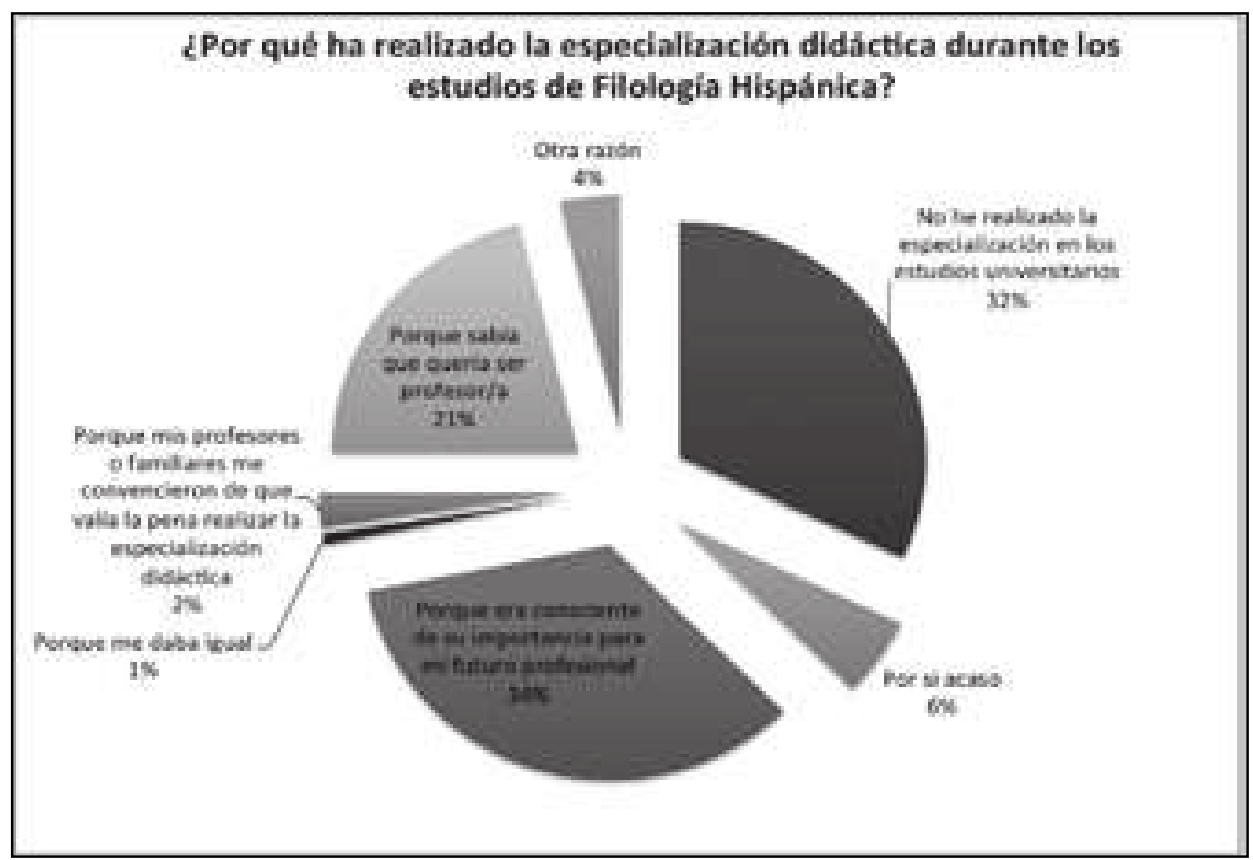

Fig. 6. Razones para realizar la especialización didáctica durante los estudios 
Hablando sobre la cuestión de la formación de los profesores de ELE que han participado en la encuesta, debemos apuntar que en su mayoría (95\%) se sienten muy preparados (57 profesores) o bastante preparados (66 profesores) para impartir clases de español (Fig. 7). Solo 5 docentes han señalado que se sienten algo o poco preparados para ejercer esta profesión. Estos datos no apoyan nuestra tesis de que los profesores de ELE en Polonia no están suficientemente preparados.

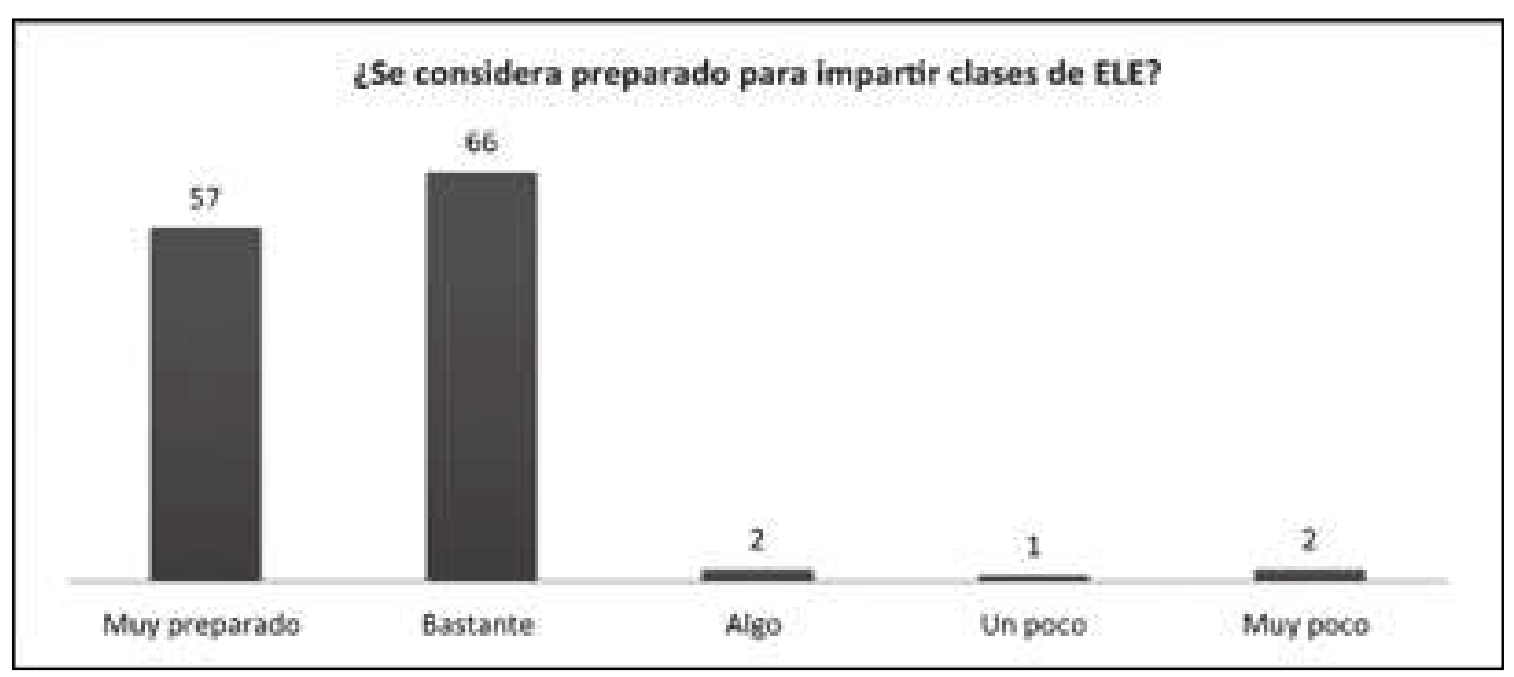

Fig. 7. Preparación de los profesores $(\mathrm{n}=128)$

También la mayoría de los docentes ha indicado que la formación que han recibido les ha ayudado en su práctica laboral (Fig. 8). Solo el $8 \%$ ha admitido que los estudios que ha realizado no le han servido de ayuda. 


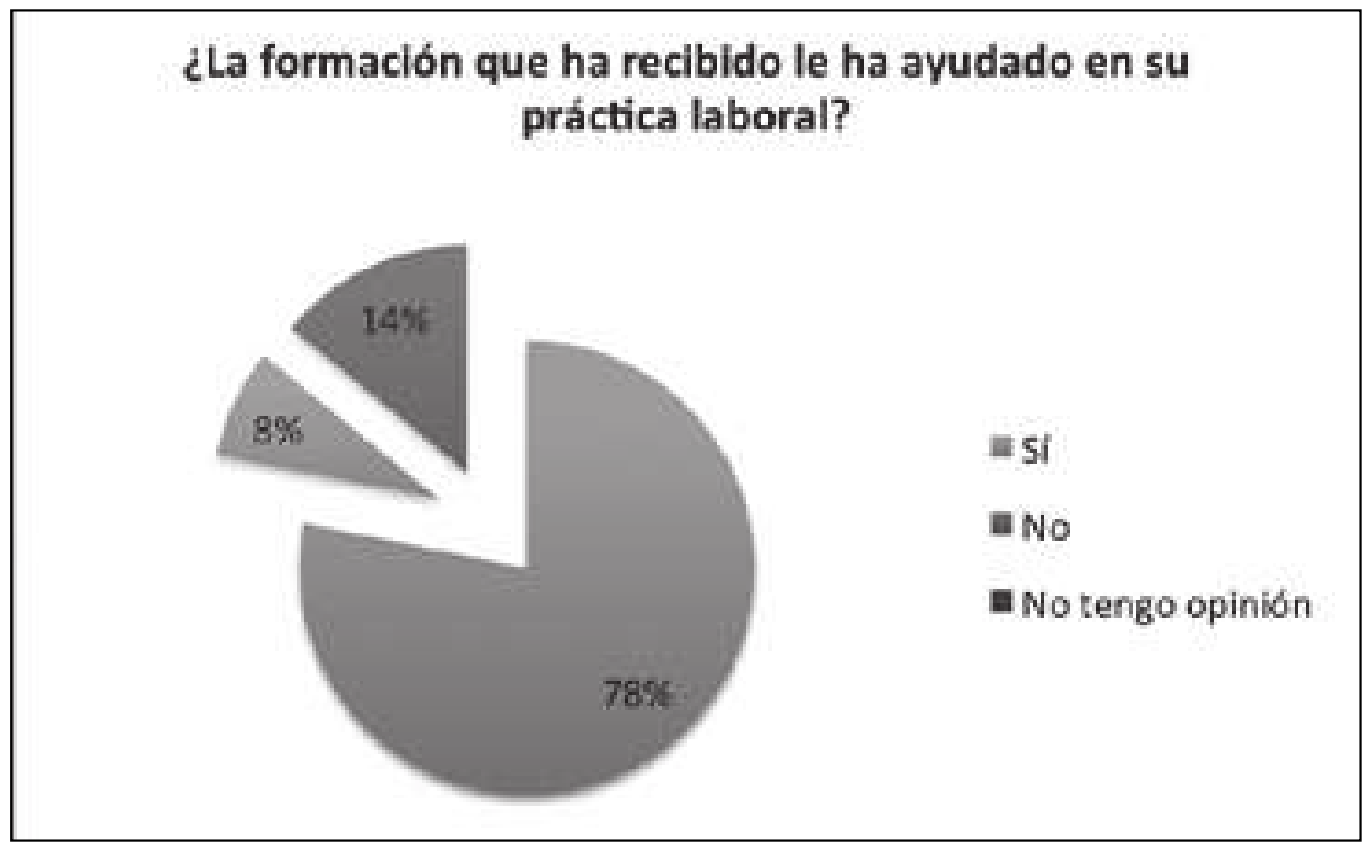

Fig. 8. Formación y práctica laboral

Los profesores podían responder también a una pregunta abierta: ¿Por qué la formación que ha recibido le ha ayudado en su práctica laboral? Abajo presentamos algunas de las opiniones:

- Me han ayudado a prepararme ante situaciones que después me he encontrado en el aula.

- Me hice una idea de qué tipo de profesora quiero ser y cómo no hay que enseñar.

- Los conocimientos de español los transmito a mis estudiantes. Los conocimientos de pedagogía también me ayudan en mi trabajo.

- Lo aprendí todo durante mis estudios: tuve buenos profesores.

- Me es más fácil preparar clases, aprendí muchos métodos y formas de trabajar, sé cómo motivar a un alumno, soy capaz de comprender y resolver problemas en el aprendizaje.

- Me ha dado una base para enseñar, pero solo la práctica hace al maestro.

- Durante mi formación universitaria aprendí español y diferentes métodos de trabajo con los estudiantes.

- Durante las clases recibíamos muchos consejos prácticos. 
- He podido conocer recursos prácticos, metodología, la parte psicológica de la enseñanza, la ley.

- En cuanto a la competencia lingüistica y pedagógica y en todos los aspectos.

- Recuerdo cómo me enseñaban a mí, qué ejercicios me gustaban y ahora los uso.

- Durante el curso de enseñanza en el EUROPROF aprendí muchas actividades útiles para las clases de ELE.

Estos ejemplos de respuestas confirman que la formación que han recibido los profesores, tanto en la universidad, como fuera de ella, les ha servido mucho en su práctica laboral. Lo que sorprende también de manera positiva es que muchos de los profesores elogian la parte práctica de esta formación y no se quejan de que han tenido pocas horas de didáctica de ELE y muchas horas de pedagogía.

También nos interesaba comprobar la opinión de los docentes acerca de la oferta didáctica que se les ofrece en el ámbito de la enseñanza de ELE en comparación con lo que ocurre con otras lenguas (Fig. 9). Debemos decir que la respuesta no nos extrañó demasiado. La mayoría $(80 \%)$ de los encuestados ha admitido que se ofrecen muy pocos talleres para ellos. Esta situación se debe, en nuestra opinión, sobre todo a la poca oferta didáctica que dedican al español las editoriales y a la falta de formadores especializados en la enseñanza de ELE en las universidades polacas ${ }^{10}$.

${ }^{10}$ El primer doctorado en Polonia dedicado a la didáctica de ELE fue defendido en 2004 por Małgorzata Spychała, el siguiente en 2013 por Renata Majewska. Los dos han sido defendidos en la Universidad Adam Mickiewicz en Poznań. 


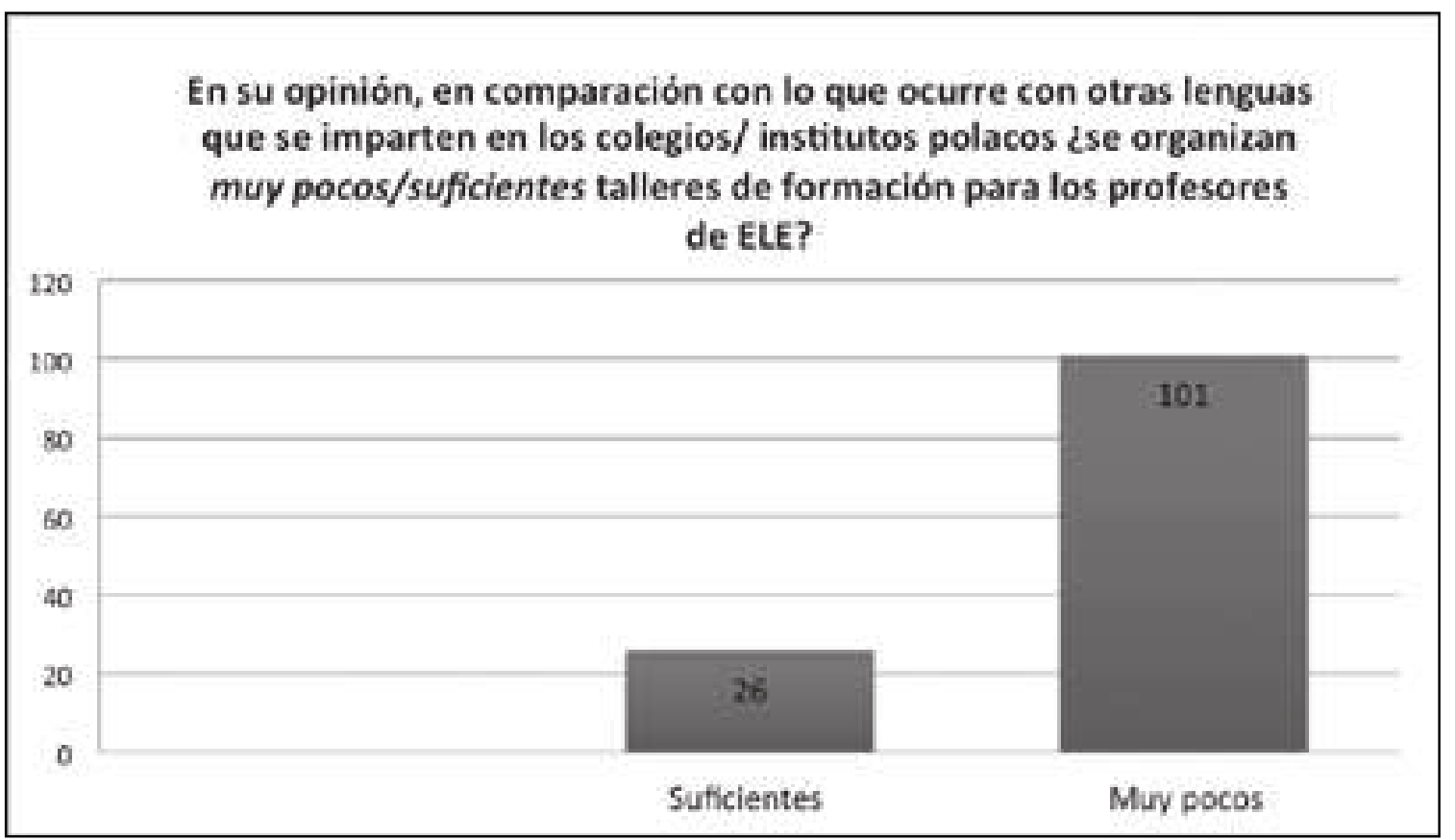

Fig. 9. Opinión sobre la oferta de talleres de formación

También preguntamos a los profesores en qué tipos de talleres les gustaría participar (Fig. 10). En este caso analizamos la media de las puntuaciones y comprobamos que no hay mucha diferencia entre las respuestas. Sin embargo, los temas que más les interesan a los profesores son: la didáctica de ELE (enseñanza de destrezas), entrenamientos culturales, o cómo trabajar con alumnos superdotados y con alumnos con dificultades de aprendizaje. Y lo que menos interesa a los profesores son los temas de pedagogía y psicología. Aunque debemos apuntar que, por ejemplo, la comunicación interpersonal, la Neurodidáctica y la disciplina en clase son temas relacionados con la pedagogía o la psicología que han conseguido mayor puntuación que psicología y pedagogía ${ }^{11}$.

${ }^{11}$ Las preguntas acerca de pedagogía y psicología son bastante generales, por eso hemos añadido las respuestas acerca de la problemática relacionada especialmente con los alumnos. 


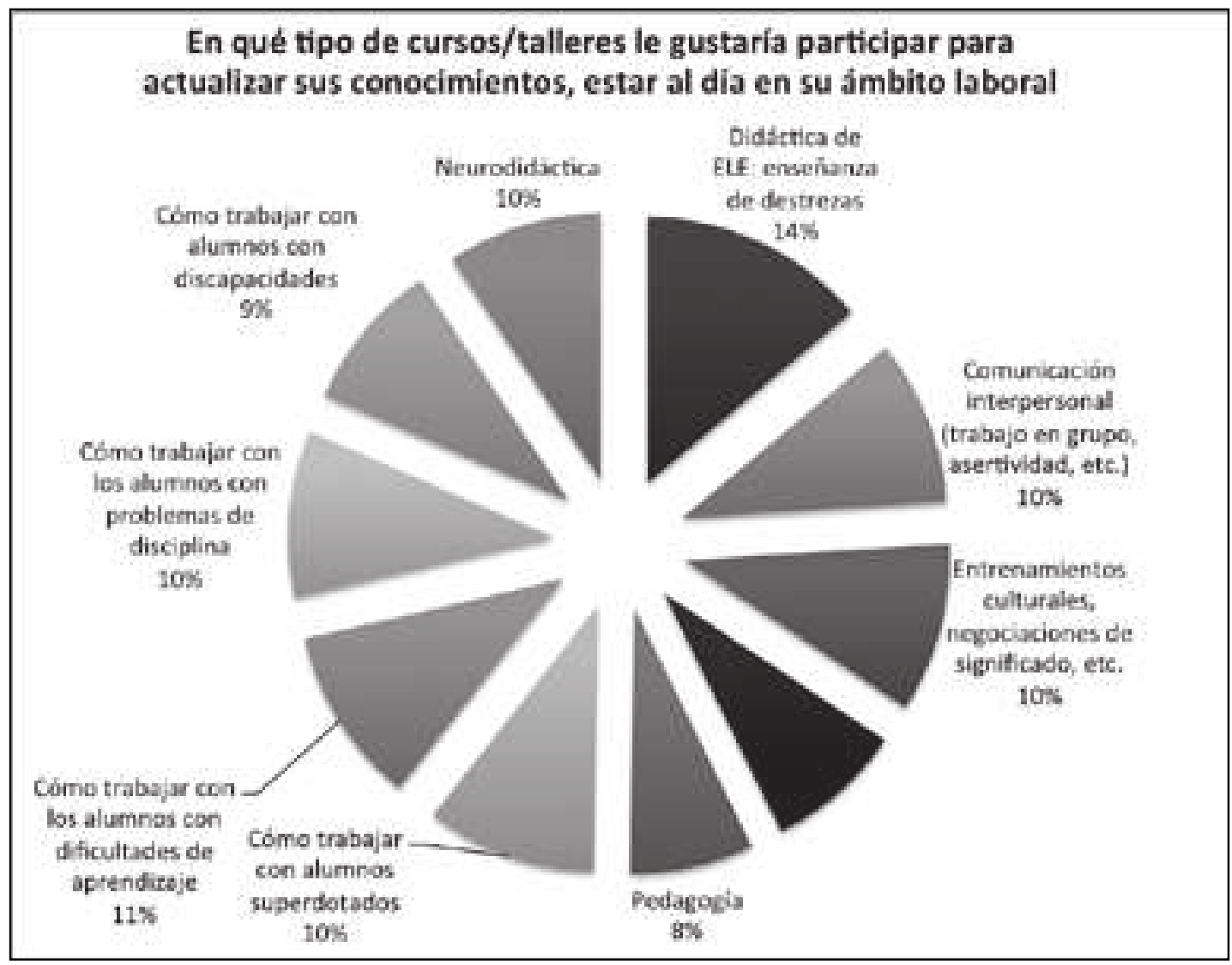

Fig. 10. Distribución de los tipos de cursos o talleres en los que les gustaría participar a los docentes para actualizar sus conocimientos y estar al día en su ámbito laboral

Los profesores también han tenido que contestar a la pregunta sobre los elementos a los que dan importancia en su práctica laboral. En este caso analizamos también el grado de su satisfacción laboral. Para presentar estos resultados hemos examinado la media de todas las respuestas y la desviación típica para las medidas de dispersión (Fig. 11). A partir de estos datos podemos afirmar que existe una cierta concordancia entre las respuestas; sin embargo, los ítems que han recibido mayor puntuación y que, por ello, son más importantes en el trabajo de los profesores de ELE en Polonia tienen que ver con el contacto con los alumnos, el poder enseñar la lengua española y el ambiente en el trabajo. Lo que sorprende es la poca puntuación que se ha dado al horario del trabajo y la remuneración. En el caso de estos ítems hay que añadir que las desviaciones típicas eran mayores, es decir, que la 
dispersión entre las puntuaciones en estas respuestas se diferenciaba más entre sí, lo que significa que no podemos afirmar con toda seguridad que para cada profesor que ha participado en la encuesta tanto el horario como el salario sean menos importantes.

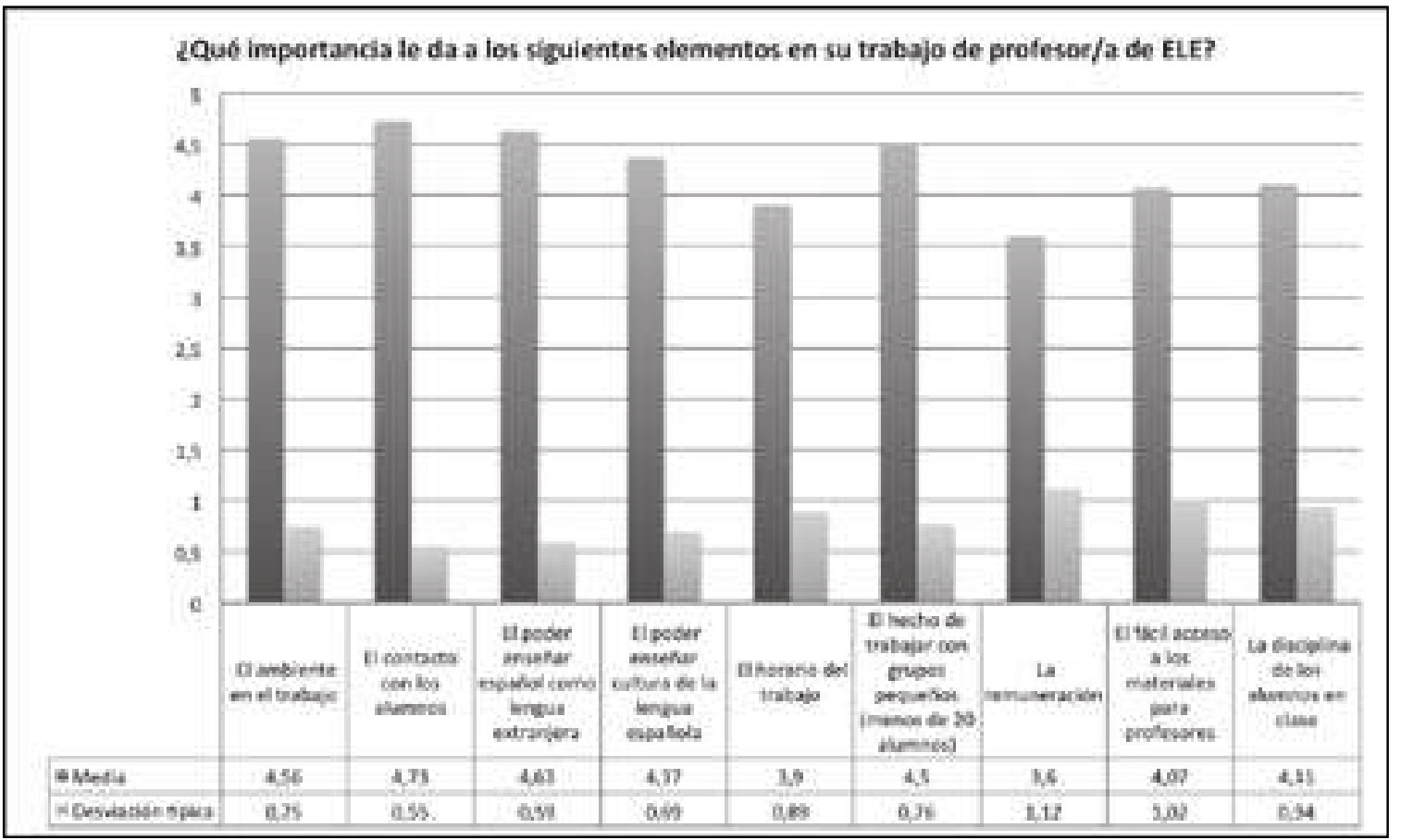

Fig. 11. Niveles de satisfacción en el trabajo $(\mathrm{n}=128)$

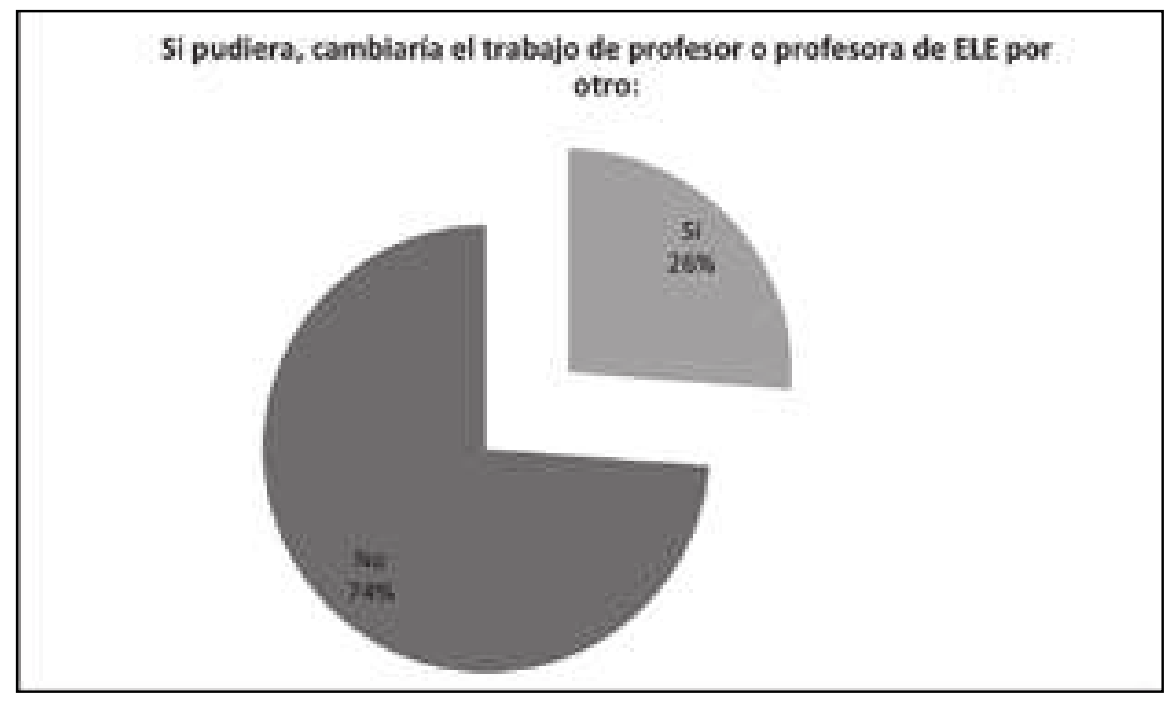

Fig. 12. Cambio de trabajo 
Finalmente, preguntamos a los profesores si cambiarían de trabajo, si pudieran. Como vemos en la figura 12, más de la mitad (74\%) de los profesores (93 encuestas) no cambiaría de trabajo, lo que revela que, en general, los profesores de ELE en Polonia se sienten satisfechos con su trabajo. Estas respuestas confirman en cierto punto los resultados obtenidos por Balches Arenas (2013: 57). En la investigación que hizo entre 19 profesores del área metropolitana de Cracovia, el 83,33\% de ellos admitió que valoraba positivamente su grado de satisfacción en el trabajo.

\section{Conclusiones}

Cada año crece la demanda del español como lengua extranjera en las escuelas primarias y secundarias en Polonia. De ahí, la necesidad de formar más profesores de ELE. El sistema educativo polaco exige que los profesores que trabajan en las escuelas preuniversitarias dispongan de las cualificaciones que regula la ley del Ministerio de Educación Nacional. En cambio, las condiciones para la formación han sido establecidas por el Ministerio de Ciencia y Enseñanza Superior.

Los resultados de nuestra investigación pueden resumirse del siguiente modo: la mayoría de los profesores de ELE que han participado en ella eran bastante jóvenes (entre 25 y 35 años) y trabajaban en un liceo (como su primer centro de trabajo). También en su mayoría eran profesores contratados (nauczyciel kontraktowy). El 68\% de ellos tenía más de 5 años de experiencia laboral y casi la mitad (47\%) ha elegido ser profesor/a de ELE porque recordaban haber querido siempre ejercer esta profesión (motivos vocacionales).

Aunque la enseñanza de ELE en Polonia es una disciplina bastante nueva, el 90\% de los profesores de ELE dispone de las cualificaciones exigidas a los profesores por la ley. E1 32\% de los encuestados ha realizado cursos de formación fuera de la universidad. E1 34\% de los profesores que ha realizado la especialización didáctica en la universidad es consciente de su importancia para su futuro profesional. El 96\% de los profesores que participaron en la investigación se considera muy preparado o bastante preparado para impartir clases de ELE. Según 
el 78\% de ellos, la formación que han recibido les ha ayudado en su práctica laboral. Hemos de admitir que estos resultados no confirman nuestra tesis acerca de que los profesores de ELE en Polonia, en su mayoría, no disponían de las cualificaciones adecuadas.

Sin embargo, muchos de los profesores opinan que, en comparación con lo que ocurre con otras lenguas que se imparten en los colegios/institutos polacos, se organizan muy pocos talleres de formación permanente para los profesores de ELE. Los docentes están interesados, sobre todo, en los talleres que actualizan los conocimientos sobre la enseñanza de las destrezas y sobre cómo trabajar con los alumnos con dificultades de aprendizaje. Otra conclusión importante atañe a la satisfacción laboral. Al respecto, podemos constatar que lo que tiene más significado para los docentes es el contacto con los alumnos y el poder enseñar español. Menor importancia tienen el horario del trabajo y la remuneración. Debemos añadir que, comparada con otros factores, la remuneración ha recibido la menor puntuación teniendo en cuenta la media: 3,6 . Aunque hay que subrayar que casi el $70 \%$ del profesorado en la escala de Likert ha señalado que les importa mucho este factor.

En conclusión, a partir de estos resultados, podemos afirmar que, en general, los profesores de ELE que trabajan en las escuelas secundarias polacas disponen de las cualificaciones laborales requeridas, se sienten preparados para ejercer esta profesión y tienen unas actitudes muy positivas hacia su labor, lo que demuestra que disfrutan de un nivel bastante alto de satisfacción laboral.

\section{Bibliografía}

ANAYA NIETO, D., SUÁREZ RIVEIRO, J. M. (2007), "Satisfacción laboral de los profesores de Educación Infantil, Primaria y Secundaria. Un estudio de ámbito nacional", Revista de Educación, 344, Madrid, pp. 217-243, [on-line] http://www.mecd.gob.es/dctm/revista-de educacion/articulosre344/re34409.pdf?documentId=0901e72b81239290, 12.04.2017.

BALCHES ARENAS, S. R. (2013), "Situación de los docentes de español/ LE en centros de enseñanza secundaria en Cracovia: aproximación a un 
análisis de expectativas y satisfacción laboral”, Studia Iberystyczne, 12, Kraków, pp. 29-74, [on-line] https://doi.org/10.12797/SI.12.2013.12.03 BRAVO, M. J., PEIRÓ, J. M., RODRÍGUEZ, I. (1996), "Satisfacción laboral”, en: Peiró, J. M., Prieto, F. (eds.), Tratado de Psicología del Trabajo. Vol. I: La actividad laboral en su contexto, Síntesis, Madrid, pp. 343-394. CHIANG VEGA, M., MARTÍN RODRIGO, M. J., NÚÑEZ PARTIDO, A. (2010), Relaciones entre el clima organizacional y la satisfacción laboral, Universidad Pontificia Comillas, Madrid.

HERMOSA RODRÍGUEZ, A. M. (2006), "Satisfacción laboral y síndrome de «burnout» en profesores de educación primaria y secundaria", Revista Colombiana de Psicología, 15, Bogotá, pp. 81-89, [on-line] https://doi. org/10.15446/rcp.

Rozporządzenie Ministra Nauki i Szkolnictwa Wyższego z dnia 17 stycznia 2012 r. w sprawie standardów kształcenia przygotowującego do wykonywania zawodu nauczyciela (Dz. U. 2012, poz. 131).

SENIOR, R. M. (2006), The Experience of Language Teaching, Cambridge University Press, Cambridge.

ZABALZA BERAZA, M. A., ZABALZA CERDEIRIÑA, M. A. (2012), Profesores y profesión docente. Entre el «el ser» y el «estar», Narcea, Madrid.

\section{Anexo 1. Encuesta realizada a los profesores de ELE en escuelas secundarias polacas}

1. Sexo: Hombre / Mujer

2. Nacionalidad:.

\section{Edad:}

- Menos de 25 años

- Entre 25 y 35 años

- Entre 36 y 45 años

- Entre 46 y 55 años

- Entre 56 y 65 años

- Más de 65 años 
4. Su primer centro de trabajo es:

- Szkoła podstawowa (primaria)

- Gimnazjum (secundaria, primer ciclo)

- Liceum (secundaria, segundo ciclo)

- Technikum (escuela de formación técnica)

- Szkoła zawodowa (escuela de formación profesional)

- Universidad o escuela superior

- Otro:

5. ¿Trabaja en un Centro Bilingüe (sekcja dwujęzyczna)?

- Sí / No

6. ¿Tiene contrato fijo como profesor/a?

- Sí / No

\section{Situación laboral:}

- Nauczyciel stażysta

- Nauczyciel kontraktowy

- Nauczyciel mianowany

- Nauczyciel dyplomowany

- Otra

8. Años de experiencia laboral como profesor/a de ELE:

- Menos de 5 años

- Entre 5 y 10 años

- Entre 10 y 20 años

- Entre 20 y 30 años

- Más de 30 años

\section{Ha elegido ser profesor/a de ELE porque:}

- recuerda haber querido siempre ejercer esta profesión

- es profesor(a) de otra asignatura y ha decidido ampliar su formación

- sus profesores o familiares le han convencido de que debe realizar la especialización didáctica en los estudios de Filología Hispánica

- no ha conseguido trabajo en otro ámbito profesional

- otro motivo: 
10. ¿Dispone de las cualificaciones (incluida la relativa a la didáctica de ELE) exigidas a los profesores para poder trabajar en escuelas preuniversitarias, de acuerdo con la ley polaca?

- Sí / No

11. Ha realizado la especialización didáctica en los estudios de Filología Hispánica:

- porque era consciente de su importancia para mi futuro profesional

- por si acaso

- porque me daba igual

- porque sabía que quería ser profesor/a

- porque mis profesores o familiares me convencieron de que valía la pena realizar la especialización didáctica

- no he realizado la especialización en los estudios

- otro motivo:

12. ¿Se considera preparado para impartir clases de ELE? Elija una respuesta (del 1 al 5)

- Muy poco

- Un poco

- Algo

- Bastante

- Muy preparado

13. ¿La formación que ha recibido le ha ayudado en su práctica laboral?

- Sí / No / No tengo opinión

- ¿Por qué "no" o por qué "sí”? Por favor, escriba su opinión.

14. En su opinión, en comparación con otras lenguas que se imparten en los colegios/ institutos polacos ise organizan muy pocos/suficientes talleres de formación para los profesores de ELE?

- Muy pocos

- Suficientes

15. ¿En qué tipo de cursos/talleres le gustaría participar para actualizar sus conocimientos, estar al día en su ámbito laboral? Valore las respuestas de 1 (no mucho) a 5 (mucho) puntos.

- Didáctica de ELE: enseñanza de destrezas

- Comunicación interpersonal (trabajo en grupo, asertividad, etc.) 
- Entrenamientos culturales, negociaciones de significado, etc.

- Psicología

- Pedagogía

- ¿Cómo trabajar con alumnos superdotados?

- ¿Cómo trabajar con alumnos con dificultades de aprendizaje?

- ¿Cómo trabajar con alumnos con problemas de disciplina?

- ¿Cómo trabajar con alumnos con discapacidades?

- Neurodidáctica

- Otros:

16. ¿Qué importancia le da a los siguientes elementos en su trabajo de profesor/a de ELE? Elija en una escala del 1 (muy poco) al 5 (mucho)

- El ambiente en el trabajo

- El contacto con los alumnos

- El poder enseñar español como lengua extranjera

- El poder enseñar la cultura de la lengua española

- El horario del trabajo

- El hecho de trabajar con grupos pequeños (menos de 20 alumnos)

- La remuneración

- El fácil acceso a los materiales para profesores

- La disciplina de los alumnos en clase

- ¿Qué otros elementos cree importantes? :

17. Si pudiera, cambiaría el trabajo de profesor o profesora de ELE por otro: - Sí / No 